\title{
Correction to: Adamts17 is involved in skeletogenesis through modulation of BMP-Smad1/5/8 pathway
}

Takeshi Oichi ${ }^{1} \cdot$ Yuki Taniguchi ${ }^{1} \cdot$ Kazuhito Soma ${ }^{1} \cdot$ Yasushi Oshima ${ }^{1} \cdot$ Fumiko Yano ${ }^{2}$. Yoshifumi Mori ${ }^{3}$. Ryota Chijimatsu ${ }^{2} \cdot$ Joo-ri Kim-Kaneyama ${ }^{4} \cdot$ Sakae Tanaka ${ }^{1} \cdot$ Taku Saito $^{1,2}$ (1)

Published online: 16 October 2019

(c) Springer Nature Switzerland AG 2019

\section{Correction to: Cellular and Molecular Life Sciences https://doi.org/10.1007/s00018-019-03188-0}

In the published article, the Fig. 4a was published incorrectly. The correct Fig. $4 \mathrm{a}$ is given below.

The original article can be found online at https://doi.org/10.1007/ s00018-019-03188-0.

Taku Saito

tasaitou-tky@umin.ac.jp

1 Sensory and Motor System Medicine, The University of Tokyo, 7-3-1 Hongo, Bunkyo-ku, Tokyo 113-8655, Japan

2 Bone and Cartilage Regenerative Medicine, Faculty of Medicine, The University of Tokyo, 7-3-1 Hongo, Bunkyo-ku, Tokyo 113-8655, Japan

3 Division of Oral Anatomy, Department of Human Development and Fostering, Meikai University School of Dentistry, 1-1 Keyakidai, Sakado 350-0283, Saitama, Japan

4 Department of Biochemistry, Showa University School of Medicine, 1-5-8 Hatanodai, Shinagawa-ku, Tokyo 142-8555, Japan 


\section{Before correction}

Fig. 4 a Immunofluorescent staining of Adamts17, Fibrillin-1 (Fbn1), and Fibrillin-2 (Fbn2) in humeral distal growth plates of WT mice at E16.5.

The inset box in the left panels indicates the location of the enlarged image shown on the right panels. Scale bars, $300 \mu \mathrm{m}$
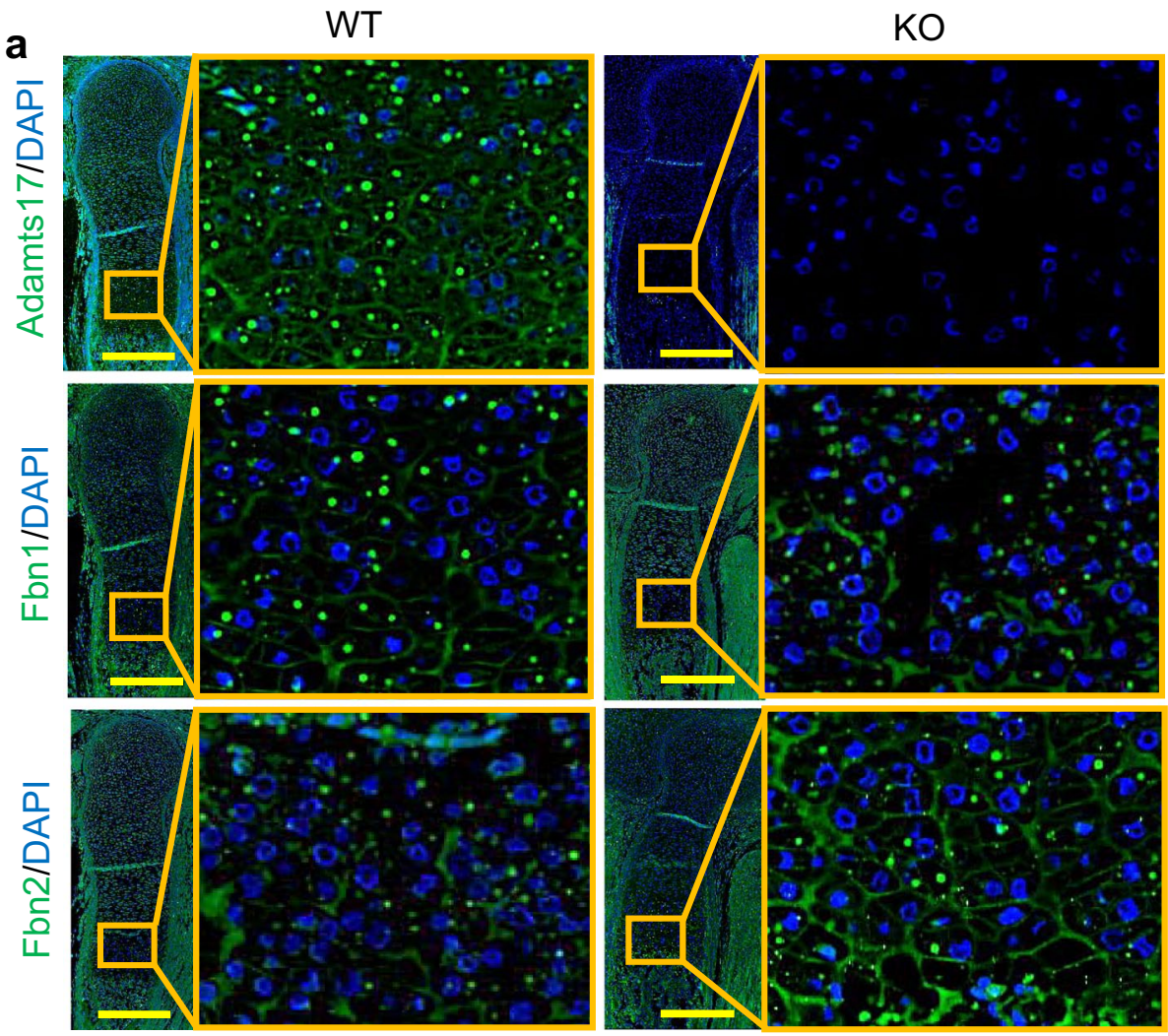

After correction

Fig. 4 a Immunofluorescent staining of Adamts17, Fibrillin-1 (Fbn1), and Fibrillin-2 (Fbn2) in the humeral distal growth plates of WT and KO mice at E16.5. The inset box in the left panels indicates the location of the enlarged image shown on the right panels. Scale bars, $300 \mu \mathrm{m}$
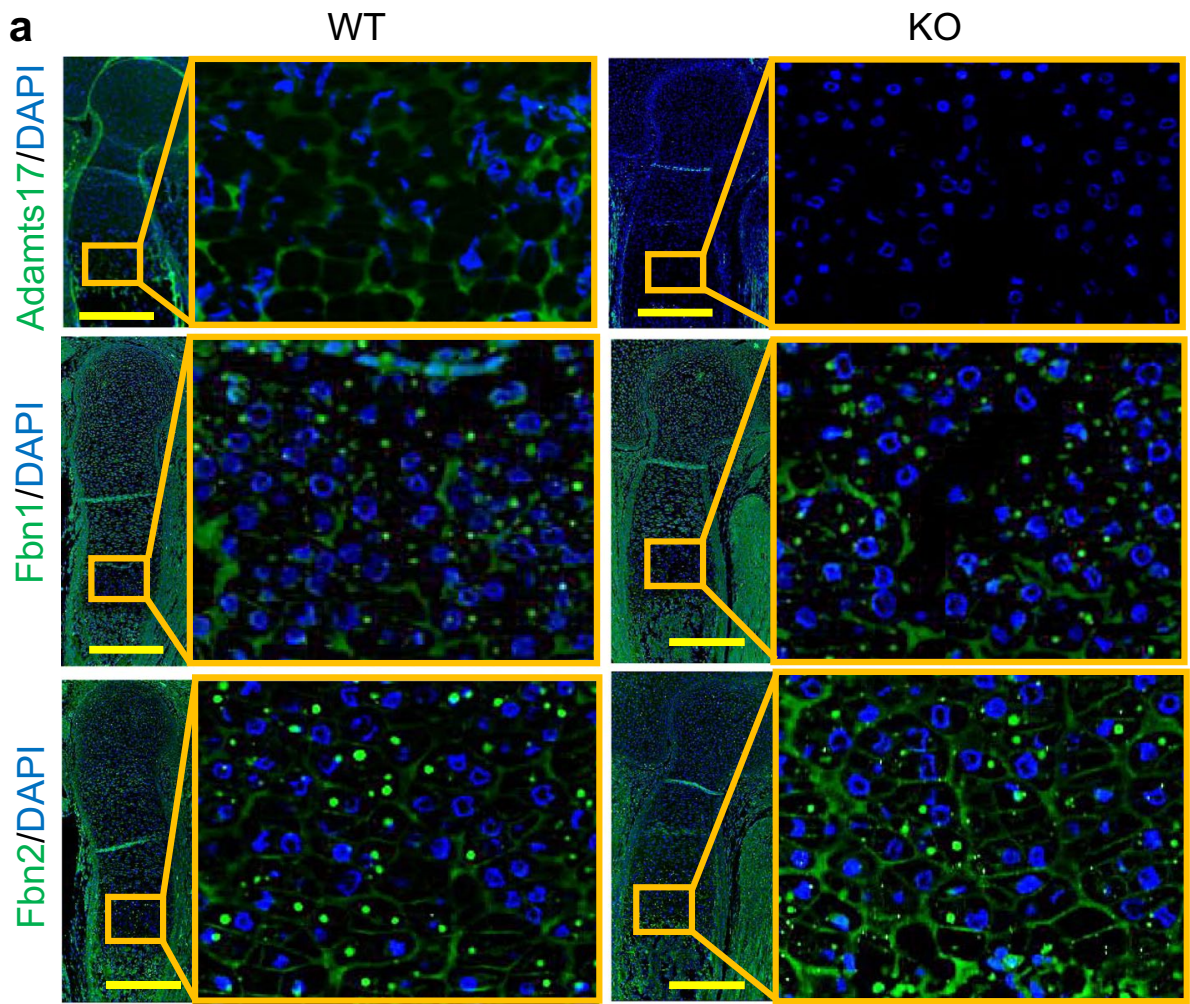